\title{
Démonstration d'un théorème sur les fonctions additives d'ensemble.
}

\author{
Par \\ W. Si erpins k i' (Varsovio). \\ (Extrait d'une lettre adressée a M. Maurice Fróchet). \\ Mon cher Oollègue.
}

Dans ma lettre du 8 avril j'ais Vous écrit que mon elève M. Saks a remarqué que le théorème de M. Franck ') se trouvo chez H. Hahn (Theorie der reellen Funlotionen, Borlin 1921, p. 404, Satz IX). Les derniers jours j'ai trouvé uno démonstration de ce théorème (sans utiliser des nombres transfiuis) qui me semble plus simple que celle de M. Hahn. Permettez moi de Vuus la comnuniquer.

Théorème. Soit une fonction d'ensembles $f$, additive et définio sur la famille additive d'ensembles $T$. Tout ensemble $W_{0}$ do la fa. mille $T$ se divise on deux ensombles $P$ et $N$, tels que PET, N\& T et

$$
\begin{array}{lll}
f(E) \geqslant 0 & \text { pour } & E \subset P, E E T \\
f(E) \leqslant 0 & \text { pour } & E \subset N, E \varepsilon T .
\end{array}
$$

Démonstration. Soit $b$ la borne supérieure de tous les nom: bres $f(E)$ pour $E C E_{0}, E \& T$ (Oomme on suit, b est un nombro fini). Il résulte de la définition du nombre b qu'il existe pour tout $n$ na. turel un ensemble $E_{n}$, tel que $E_{n} \subset E_{0}, E_{n} \varepsilon^{\prime} T^{\prime}$ et

$$
f\left(E_{n}\right)>b-\frac{1}{2^{n}}
$$

1) V. ce volume, p. 257. 


\section{Posons}

où

et posons

(6)

$$
P=\lim E_{n}=H_{1} H_{2} H_{3} \ldots
$$

$$
H_{n}=E_{n}+E_{n+1}+E_{n+2}+\ldots \quad(n=1,2,3, \ldots)
$$

$$
N=E_{0}-P
$$

je dis que $P$ et $N$ sout les ensembles cherchés.

En effet, admettons que la formule (1) ne subsiste pas. Il existe alors un ensemble $E$, tel que

$$
E \subset P, E \varepsilon T, \text { et } f(E)=-\nu<0 .
$$

Soit $n$ un nombre naturel, tel que

$$
\frac{1}{2^{n-1}}<\nu
$$

D'après (7) et (4), nous avons $E \subset H_{n}$, donc, d'après (5):

$$
\begin{gathered}
E=H_{n} E=E_{n} E+E_{n+1}\left(E-E_{n}\right)+E_{n+2}\left[E-\left(E_{n}+E_{n+1}\right)\right]+\ldots=, \\
=E_{n}^{\prime}+E_{n+1}^{\prime}+E_{n+2}^{\prime}+\ldots,
\end{gathered}
$$

où $E_{n+k}^{\prime}(k=0,1,2, \ldots)$ sont des ensembles disjoints et

$$
E_{n+k}^{\prime} \subset E_{n+k}, E_{x+k}^{\prime} \varepsilon T, \text { pour } k=0,1,2, \ldots
$$

Nous avons done:

$$
f(E)=f\left(E_{n}^{\prime}\right)+f\left(E_{n+1}^{\prime}\right)+f\left(E_{n+2}^{\prime}\right)+\ldots
$$

D'après (3) nous avons

$$
f\left(E_{n+k}\right)>b-\frac{1}{2^{n+k}} \quad(k=0,1,2, \ldots) .
$$

et, d'après (9): $E_{n+k}=E_{n+k}^{\prime}+\left(E_{n+k}-E_{n+k}^{\prime}\right),\left(E_{n+k}-E_{n+k}^{\prime}\right) \varepsilon T$, donc, d'après (11):

$$
f\left(E_{n+k}^{\prime}\right)+f\left(E_{n+k}-E_{n+k}^{\prime \prime}\right)=f\left(E_{n+k}\right)>b-\frac{1}{2^{n+k}}, \quad(k=0,1, \ldots)
$$

or, d'après la définition du nombre $b$, nous avons

done, d'après (12):

$$
f\left(E_{n+k}-E_{n+k}^{\prime}\right) \leqslant b
$$

$$
f\left(E_{n+k}^{\prime}\right)>-\frac{1}{2^{n+k}} \text { pour } k=0,1,2, \ldots
$$

et (10) donne:

$$
f(E)=\sum_{k=0}^{\infty} f\left(E_{n+k}^{\prime}\right)>-\sum_{k=0^{-}}^{\infty} \frac{1}{2^{n+k}}=-\frac{1}{2^{n-1}},
$$


264 W. Sierpiński: Fonctions additives d'ensemble.

donc, d'après (8):

contrairement à (7).

$$
f(E)>-\nu,
$$

Nous avons done démontré la formule (1).

Admettons maintenant que la formule (2) ne subsiste pas. II existe alors un ensemble $E$ tel que $E \subset N, E \varepsilon T^{\prime}$ et

$$
f(E)=\pi>0
$$

Soit $n$ un nombre naturel, tel que

$$
\frac{1}{2^{n-1}}<\pi
$$

D'après (6), (4) et (5), nous trouvons sans peine

$$
N \subset\left(E_{0}-E_{n}\right)+\left(E_{0}-E_{n+1}\right)+\left(E_{0}^{\prime} \ldots E_{n+2}^{\prime}\right)+\ldots,
$$

done, d'après $E \subset N, E \varepsilon T$ :

$$
E=E_{n}^{\prime}+E_{n+1}^{\prime}+E_{n+1}^{\prime}+\ldots
$$

où $E_{n+k}^{\prime}(k=0,1,2, \ldots)$ sont des ensembles disjoints et tels que

$$
E_{n+\star}^{\prime} \subset\left(E_{0}-E_{n+k}\right), \quad \text { et } \quad E_{n+k}^{\prime} \varepsilon T \quad(k=0,1,2, \ldots) \text {. }
$$

D'après (16) nous avons

$$
E_{n+k}^{\prime} E_{n+k}=0, \quad E_{n+k}^{\prime}+E_{n+k} \subset E_{0}^{\prime}
$$

done, d'après la définition du nombre $b$ :

$$
f\left(E_{n+k}^{\prime}\right)+f\left(E_{n+k}\right)=f\left(E_{n+k}^{\prime}+E_{n+k}^{\prime}\right) \leqslant b,
$$

danc, d'après (11):

$$
f\left(E_{n+k}^{\prime}\right)<\frac{1}{2^{n+k}}, \text { pour } k=0,1,2 \ldots
$$

ce qui donne, d'après (15):

done, d'après (14):

$$
f(E)=\sum_{k=0}^{\infty} f\left(E_{n+k}^{\prime}\right)<\sum_{k=0}^{\infty} \frac{1}{2^{n+k}}=\frac{1}{2^{n-1}},
$$

contrairement ì (13).

$$
f(E)<\pi
$$

La formule (2) est aiusi établie et lo thérorèmo oat démontré.

Varovie, lo 12 Mai 1928. 